\title{
CONTAMINATION AND CURRENT PRACTICE IN DECONTAMINATION OF NEBULISERS IN VENTILATED PATIENTS
}

\begin{abstract}
Objectives: To examine the incidence of contamination and current practice of decontamination of nebulisers after use within a ventilator circuit, in public- and private sector intensive care units (ICUS) in Johannesburg; to assess the presence of and adherence to a decontamination protocol in these ICUs and to identify which practices were associated with lower or no bacterial growth.

Methods: A cross-sectional study design was used which included a semistructured interview with the ICU manager and an audit of current nebuliser practice. Nebulisers that were identified in the interview were swabbed and streaked on blood agar plates (BAPS). BAPs were incubated and assessed for bacterial colonisation, number of colony forming units (CFUS) and number of different species of CFUs present.
\end{abstract}

\begin{tabular}{|l|}
\hline Ellis A, MSc Physiotherapy ${ }^{1} ;$ \\
Van Aswegen H, PhD \\
Roos R, MSc Physiotherapy \\
'; \\
P Becker, PhD ${ }^{2}$ \\
1 Department of Physiotherapy, \\
Faculty of Health Sciences, University of \\
the Witwatersrand, Johannesburg. \\
2 Biostatistics Unit, Medical Research \\
Council, Pretoria.
\end{tabular}

Results: Two hundred and sixty nine ICU beds were surveyed over a two month period resulting in 45 nebulisers used within a ventilator circuit that could be tested. The majority (93\%) were single-use jet nebulisers, all were being re-used and $52 \%$ presented with contamination. None of the ICUs had a nebuliser decontamination protocol in place. Contaminated nebulisers that were stored in a sterile drape had significantly higher concentrations of bacterial growth ( $p=0.03$ ).

Conclusion: The rate of colonisation of re-used jet nebulisers is high. Nebuliser decontamination protocols are urgently needed.

KEY WORDS: NEBULISERS; VENTILATOR; CONTAMINATION; DECONTAMINATION PROTOCOL

\section{INTRODUCTION}

Nebulisation is a widely accepted and effective form of medication administration both inside and outside of the intensive care unit (ICU) (Dhand 2008). Nebulisation is the aerosolisation of a liquid in order to allow deposition of particles directly into the respiratory tract resulting in decreased systemic side effects and improved local effect. The type of device used during nebulisation

\section{Correspondence Author:}

$\mathrm{H}$ van Aswegen

Associate-Professor,

Department of Physiotherapy,

Faculty of Health Sciences,

University of the Witwatersrand,

7 York Road

Parktown

2193

Johannesburg

Email: helena.vanaswegen@wits.ac.za affects particle size, and thus depth of penetration. Jet nebulisers are commonly used in ICU, followed by ultrasonic and more recently vibrating mesh nebulisers (VMN), all of which may be connected into the ventilator circuit (Fink 2009; Robinson, Athota and Branson 2009; Dhand 2008). Ventilatorassociated pneumonia (VAP) a condition that develops in ventilated patients after more than 48 hours, represents $86 \%$ of hospital acquired pneumonias and has a 30\% mortality (Valencia and Torres 2009; Rotstein et al 2008). A contaminated nebuliser within a ventilator circuit potentially can deliver pathogens deep into the lower respiratory tract (Dhand 2008; Ball et al 2005).

Physiotherapists and nursing sisters in ICU regularly use nebulisation to administer medication such as bronchodilators and/or mucolytic drugs to intubated patients. The current practice of storage of a nebuliser in Johannesburg
ICUs involves storing the nebuliser in either a non-sterile glove or sterile drape next to the patient's bed until the next dosage of medication is to be administered. Residual medication is often left in the chamber when stored. It has been observed that due to cost constraints devices marked as singleuse are being used as single-patient-use devices. There is no information on the incidence of contamination of singleuse nebulisers that are re-used within a ventilator circuit or documentation of which decontamination methods are currently in place. The aims of this study were to examine the incidence of contamination and current practice of decontamination of nebulisers after use within a ventilator circuit in ICUs in Johannesburg; to assess the presence of and adherence to a decontamination protocol in these ICUs and to identify which practices are associated with low or no bacterial growth. 


\section{METHOD}

\section{Study design}

A cross-sectional study design was used. The ICU staff were blinded as to the nature of the study until the time of interview, in order to assess current practice without influencing the staff's approach to nebuliser use and decontamination. All ICUs of government$(n=4)$ and private $(n=10)$ hospitals in Johannesburg, South Africa were considered for inclusion in this study. The hospitals that were approached were selected on the basis of geographical location in order to obtain a representative sample. Within the private hospital group, representation of the three main hospital groups (Netcare, Life Healthcare and Medi-Clinic) was present. The nebulisers that were in the ICU at the time of audit were selected for assessment. Nebulisers had to have been used within a ventilator circuit for patients with artificial airways such as endotracheal or tracheostomy tubes.

\section{Ethical considerations}

Permission was obtained from the University of the Witwatersrand Human Research Ethics Committee to conduct the study and from the hospital manager/CEO of the participating hospitals to access the ICUs of that hospital and to approach the ICU staff. The staff of the ICUs that were assessed was given information sheets regarding the nature of the study and the information to be collected but as they were not being directly observed they did not have to give consent for the assessment of the nebulisers in the unit. There was no direct contact with patients during this study.

\section{Data collection tools}

An audit tool which consisted of two sections was used to collect data. The first section was used to collect the basic information of the unit on the day of audit e.g. number of ventilated patients in the unit and the second section was used to record the individual assessment of each included nebuliser. The swabs used to swab the nebulisers were cotton tipped, wooden handled and autoclaved in the University of the Witwatersrand Pharmaceutical Microbiological Laboratory (PML) before use. Sheep's blood agar was used to culture the collected swabs. The blood agar plates (BAP) were pre-prepared at the PML and plate diameter was $6.5 \mathrm{~cm}$. Plates were transported in a cardboard box within a cooler box, in order to reduce the fluctuations in temperature during transport to the various hospitals. The first author was trained by staff from PML during a pilot study to follow the correct swabbing procedure as described in the swabbing protocol for PML and in the assessment of BAP after incubation.

\section{Sample size calculation}

The sample size for the main study was calculated based on the number of ICU beds in Gauteng $(n=1938)$ (Bhagwanjee and Scribante 2007). The alpha level was set at $p<0.05$ and the estimated prevalence of ventilated patients at $50 \%$. The frequency of the use of nebulisers in a ventilated population in Johannesburg is an unknown variable, and could not be used for the power calculation. The number of ICU beds to be surveyed for this study was set at 278 (power level at $80 \%$ ).

\section{Procedure}

Once consent was obtained from the hospital manager/CEO, the unit manager of each ICU within that particular hospital (public- and private sectors) was approached and an appropriate day for the audit was agreed upon. On the day of assessment the basic information of the ICU (number of beds, number of patients ventilated and on nebulisation, nebuliser decontamination practice) was obtained through a semi-structured interview with the unit manager or shift leader. The unit manager/shift leader then identified which beds had patients who were ventilated. Each bedside was checked by the first author together with the unit manager/shift leader to establish the presence of a nebuliser and all nebulisers that met the inclusion criteria were then assessed. The first author observed how each nebuliser was stored and tested for any evidence of decontamination.

Each nebuliser was swabbed by the first author to assess for contamination according to the following protocol: the first author washed her hands and donned nitrile powder free gloves; the nebuliser was removed from the bedside; placed on a clean even surface close to the bedside and if the oxygen tubing was attached it was removed to aid with the swabbing procedure. The outside of the nebuliser was wiped with isopropyl alcohol to ensure that there was no external contamination of the nebuliser. The nebuliser was opened and placed on clean gauze swabs. The base plate was removed from the nebuliser, ensuring that the sides of the chamber were not touched. Two sterile swabs were dipped into the residual solute within the reservoir of the nebuliser. If there was less than $2 \mathrm{ml}$ of liquid in the nebuliser, $2 \mathrm{ml}$ of sterile water was added to the reservoir. The swabs were immediately streaked across BAP. The plates were then labelled and returned to the transport box. The area was cleaned and the nebuliser reassembled and returned to the patient's bedside, in its original position and condition.

Each nebuliser assessed resulted in two BAP. The BAP were transported back to the PML. One plate was incubated at 25 degrees Celsius $\left({ }^{\circ} \mathrm{C}\right)$ for seven days to monitor if any fungal contamination was present. The second plate was incubated at $37^{\circ} \mathrm{C}$ for 24 hours in order to assess for possible bacterial contamination. On completion of the required incubation time the BAP were assessed. The number of different colonies was identified and then the number of colony-forming units (CFU) for each type of colony was counted using visual inspection. Categorisation of CFUs is shown in Table 1.

For concentration of CFUs, if there were multiple species present, the species with the highest CFU count was considered for ranking. CFUs were only differentiated by their visual characteristics when determining different species. No other testing was done to determine which species were present in the BAPs. The BAPs were double counted, and the verified result documented. Photographs of the BAPs were archived in case the need for reassessment of a BAP arose.

\section{Statistical Analysis}

Data were captured on Microsoft Excel spreadsheets. Categorical variables were summarised as numbers and per- 
centages. Continuous variables were summarised as means, medians and standard deviations. Fisher's exact test and t-test were used for hypothesis testing to determine practices associated or not associated with bacterial growth in nebulisers. All testing was performed at the $p<0.05$ level of significance. Data analysis was performed with STATA 10 software package.

\section{RESULTS}

A total of 13 hospitals were approached to participate in the study. Six private and three government sector hospitals participated $(n=9)$. These hospitals represented 21 ICUs. A total of 269 ICU beds were surveyed over a two month period. This sample was slightly lower than the calculated sample as not all hospitals gave consent for participation in the study. A total of 45 nebulisers that had been used within a ventilator circuit were identified in the sample. The average number of beds per unit was $14(\mathrm{SD} \pm 6.85)$ and the average number of ventilated patients per unit was $4(\mathrm{SD} \pm 2.94)$. Of the patients in ICU $(n=269), 17 \%$ were ventilated and received nebulisation. The type of nebulisers used and the medication found in the nebulisers are summarised below in Table 2.

Single-use jet nebulisers were mostly used: government sector, $\mathrm{n}=5(62.5 \%)$ and private sector, $\mathrm{n}=37(100 \%)$. All nebulisers assessed $(n=45)$ were being re-used and $93 \%$ of all nebulisers assessed were not being used in accordance with the manufacturer's recommendations, as they were marked as single-use devices. There were no VMNs found in the ICUs surveyed. The administration of bronchodilator medication as well as mucolytics ranked the highest for the type of medication used in the nebulisers $(76 \%$ and $21 \%$ respectively). Nebulisers were occasionally used for administration of corticosteroids and Lasix. The median time in which the nebulisers that were tested had been stored since nebulisation, was four hours.

Table 3 summarises decontamination of nebuliser practice in the ICUs surveyed.

Most frequently [82\% ( $\mathrm{n}=37)]$ nebulisers were stored wet and 93\% $(n=42)$

Table 1: Categorisation of Colony Forming Units

\begin{tabular}{|l|l|l|l|}
\hline \multicolumn{2}{|c|}{ Concentration of CFUs } & \multicolumn{2}{|c|}{ Number of different colonies } \\
\hline 0 & No Growth & 1 & 1 species \\
\hline 1 & $1-2$ CFUs of 1 species & 2 & 2 species \\
\hline 2 & $3-100$ CFUs of 1 species & 3 & 3 species \\
\hline 3 & $>100$ CFU of 1 species & 4 & 4 species \\
\hline
\end{tabular}

Table 2: Nebuliser Type and Medication used in Nebulisers

\begin{tabular}{|l|c|}
\hline \multicolumn{1}{|c|}{ Type of Nebuliser } & $\mathrm{n}(\%)$ \\
\hline Single-use Jet Nebuliser & $5(62.5 \%)$ \\
• Government sector $\quad$ Private sector & $37(100 \%)$ \\
Single-patient-use Jet Nebuliser & $1(12.5 \%)$ \\
$\bullet \quad$ Government sector & $0(0 \%)$ \\
Single-patient-use Autoclavable $\quad$ Private sector & \\
Ultrasonic Nebuliser & \\
$\bullet \quad$ Government sector & $2(25 \%)$ \\
Private sector & $0(0 \%)$ \\
\hline Medication used in Nebulisers & $\mathrm{n}(\%)$ \\
\hline Combivent & $12(26.7 \%)$ \\
Saline & $8(17.8 \%)$ \\
Atrovent and Saline & $5(11.1 \%)$ \\
Atrovent & $5(11.1 \%)$ \\
Atrovent and Berotec & $5(11.1 \%)$ \\
Duolin & $4(8.9 \%)$ \\
Unknown medication & $2(4.4 \%)$ \\
Atrovent and Pulmicort & $1(2.2 \%)$ \\
Bisolvon & $1(2.2 \%)$ \\
Doulin and Saline & $1(2.2 \%)$ \\
Lasix & $1(2.2 \%)$ \\
\hline
\end{tabular}

Table 3: Current Methods of Nebuliser Decontamination

\begin{tabular}{|l|c|c|}
\hline \multirow{2}{*}{} & \multicolumn{2}{|c|}{$\mathbf{n}(\%)$} \\
\cline { 2 - 3 } & Yes & No \\
\hline Nebuliser discarded after use & $0(0 \%)$ & $45(100 \%)$ \\
\hline Nebuliser stored dry & $8(17.8 \%)$ & $37(82.2 \%)$ \\
\hline If dry, solute visible in chamber & $7(87.5 \%)$ & $1(12.5 \%)$ \\
\hline Stored in a glove & $19(42.2 \%)$ & $26(57.8 \%)$ \\
\hline Stored in a sterile drape & $17(37.8 \%)$ & $28(62.2 \%)$ \\
\hline Stored open to the environment & $5(11.1 \%)$ & $40(88.9 \%)$ \\
\hline Stored removed from $\mathrm{O}_{2}$ tubing & $3(6.7 \%)$ & $42(93.3 \%)$ \\
\hline Autoclaved & $0(0 \%)$ & $45(100 \%)$ \\
\hline
\end{tabular}

nebulisers were stored connected via oxygen tubing to the flow meter or ventilator. Bacterial growth was found in 23 of the nebulisers that were swabbed which represented $51.1 \%$ of nebulisers used within a ventilator circuit. Isolation of the batch of singleuse nebulisers that had been re-used $(n=42)$ as a separate group from the single-patient-use nebulisers, revealed a rate of contamination of $52.4 \%$ $(n=22)$. Between two and four CFUs 


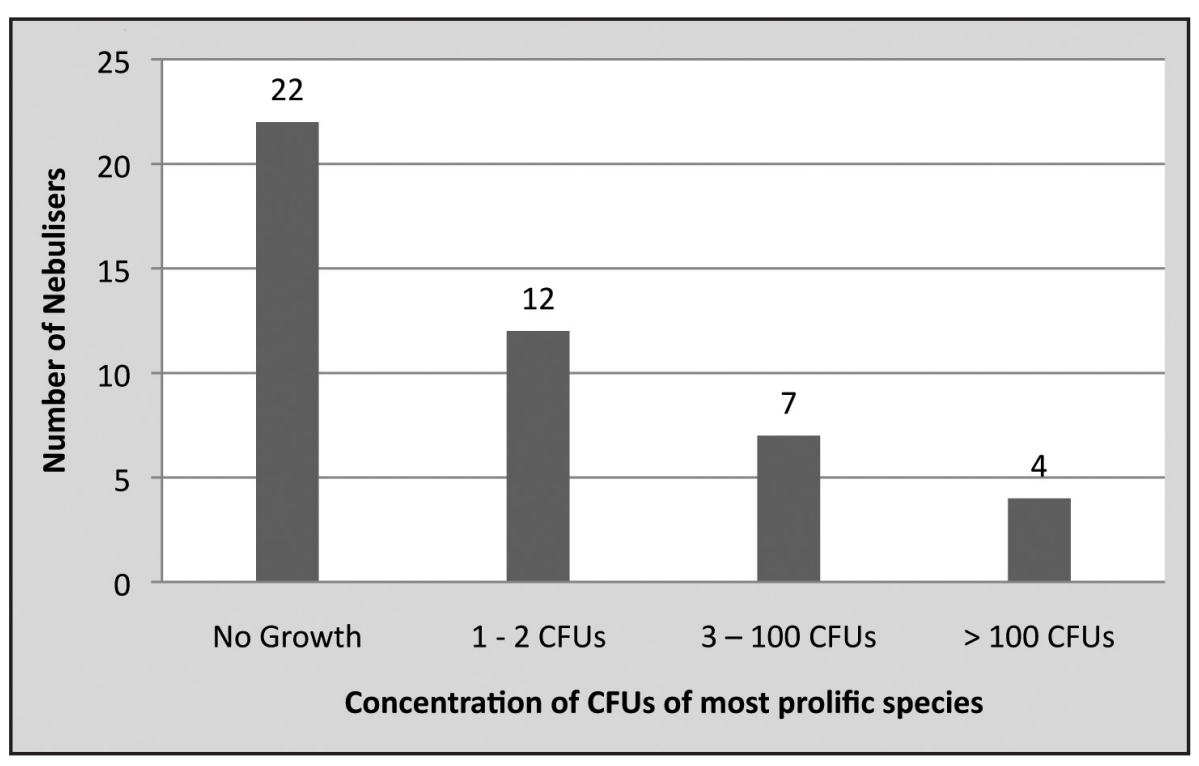

Figure I: Concentration of colony forming units of most prolific species

Table 4: Factors not associated with Rate of Contamination or Bacterial Growth in Nebulisers

\begin{tabular}{|c|c|}
\hline \multicolumn{1}{|l|}{} & p-value \\
\hline Rate of contamination of nebuliser & 0.66 \\
- $\quad$ Nebuliser manufacturer & 0.39 \\
\hline Bacterial growth inside contaminated nebuliser & 0.46 \\
- $\quad$ Storage of nebuliser wet or dry & 0.77 \\
- Storage of nebuliser in a latex glove & 1.00 \\
$\quad$ Storage of the nebuliser open to the & 0.23 \\
$\quad$ Storage of nebulizer connected to oxygen & 0.57 \\
$\quad$ tubing & 0.57 \\
\hline
\end{tabular}

were identified on $10(44 \%)$ of the 23 nebulisers with bacterial growth. Eleven ( $48 \%$ ) of the 23 contaminated nebulisers had more than three CFUs of most prolific species (Figure 1).

None of the ICUs assessed had a nebuliser decontamination protocol. Nebulisers that were stored in a sterile drape and had bacterial growth, had higher CFU concentrations than those that were not stored in a drape and had bacterial growth $(p=0.03)$. A factor that therefore enhanced bacterial growth was storage in a sterile drape. Table 4 summarises the factors not associated with rate of contamination of or bacterial growth in nebulisers.

One nebuliser was visibly soiled with secretions. This nebuliser ranked second for concentration and fourth for number of different species of CFU noted. Two of the nebulisers in one specific ICU were left with $1 \ell /$ min of oxygen running through and were stored in paper autoclave bags. Both nebulisers were dry on assessment, but had residual solute in them. Interestingly, neither of these nebulisers had bacterial growth.

\section{DISCUSSION}

This study highlights several important findings which include the fact that the majority of nebulisers used within a ventilator circuit in Johannesburg were single-use devices which were being re-used. Secondly, more than half of the single-use jet nebulisers that were re-used had bacterial growth, and contaminated nebulisers that were stored in sterile drapes had higher bacterial concentrations than those that were contaminated but not stored in a drape. Of concern was that none of the ICUs had a nebuliser decontamination protocol in place.

The re-use of single-use jet nebulisers is in direct contravention to manufacturers guidelines. Similar to our findings Allan et al. (2004) reported that devices intended for single-use were re-used on the same patient with little or no decontamination or reprocessing between nebulisations. The rationale behind the reprocessing of singleuse nebulisers was the high cost involved in using a new device with each nebulisation. Cost constraints may potentially influence decisionmaking regarding the type of nebulisers used in the ICU towards those that are less expensive, disposable singleuse devices; however the cost of healthcare-associated infections far outweighs this potential cost difference. Importantly, manufacturers' guidelines for jet nebulisers are for cleaning only. The manufacturers themselves issued statements indicating that their cleaning guidelines do not sterilize the device and that microbial contamination of the device may persist after a cleaning procedure (Edwards 2001). When staff re-use devices that were labelled as single-use by the manufacturers they take the legal responsibility of this altered use on themselves. Should an adverse event arise for a patient due to this altered usage, the healthcare provider will be held liable and not the manufacturer (Allan et al 2004). The influence of re-use of single-use devices on effectiveness of drug delivery is unknown. Thus physiotherapists and nurses are potentially putting themselves at risk for litigation every time they reuse a device that is marked as single-use by the manufacturer. A solution to this situation would be the introduction of evidence-based protocols for the re-use and decontamination of such devices.

More than half of the single-use jet nebulisers that had been re-used had bacterial colonisation. This figure has to be viewed in the context of zero contamination if the nebulisers were discarded after use and a new nebuliser used for each nebulisation. The use of metered-dose inhalers 
(MDIs) to administer medication into a ventilator circuit may overcome some of the infection control issues posed by contaminated jet nebulisers. In this study bronchodilators were predominantly used for nebulisation. These drugs are readily available as MDIs. The use of VMNs may also address the problem of re-using single-use jet nebulisers. The reservoir of VMNs is separated from the ventilator circuit which potentially reduces the risk of contamination and colonisation. The VMN is electronically triggered at a specific phase of inspiration and does not introduce extra flow into the circuit, which may ultimately make this device more effective than jet nebulisers (Fink 2009; Dhand 2008).

Nebulisers that had bacterial growth and were stored in a sterile drape had higher concentrations of bacterial growth than nebulisers that were stored either in a non-sterile glove or open to the environment. No supporting literature for the use of sterile drapes as a method of storage of nebulisers is available. The drape itself may not have been the source of contamination, but it seems that once a contaminated nebuliser was stored within a drape, the bacteria multiplied more prolifically than in nebulisers that were stored in other ways. The dark environment of the drape may contribute to bacterial growth, as light inhibits the growth of bacteria such as Pseudomonas Aeruginosa (Sagripanti et al 2013). The sterile drapes may stay with a patient for their entire period of ventilation, whereas latex gloves are more easily discarded and replaced.

Two of the nebulisers that were stored with a low flow rate of oxygen ( $1 \ell / \mathrm{min})$ running through them did not have any bacterial growth. This could have acted in one of two ways to inhibit bacterial growth within the nebuliser. Firstly the flow of oxygen could have dried the nebuliser faster. Although there is no statistical evidence in this study to show that storing nebulisers wet or dry affects bacterial growth, it has been documented elsewhere that bacteria such as Pseudomonas Aeruginosa thrive in moist environments (Anaissie, Penzak and Dignani 2002). It is reasonable to suppose that nebulisers that are stored dry are less likely to encourage bacterial growth of certain strains. Secondly high concentrations of oxygen within a nebuliser chamber reduce bacterial growth of Pseudomonas Aeruginosa (Schobert and Tielen 2010). The application of oxygen for a short period through the nebuliser before storage may assist in the inhibition of bacterial growth within the nebuliser. Both these nebulisers were stored in paper bags (obtained after a sterile pack was opened). Paper has a greater absorbency than latex gloves. Latex gloves however, trap water and solute within the nebuliser and in so doing hamper the drying process.

The first author was unable to collect data on how long each nebuliser had been in use prior to swabbing. None of the nebulisers had a documented date of first use. It is important that ICU staff who continue to re-use single-use nebulisers create a protocol for their unit which highlights information including number of nebulisations a nebuliser may complete before it should be discarded; method of cleaning the nebuliser after use; recommended storage procedure; specific criteria that indicate when a nebuliser should be discarded; method of recording usage of each nebuliser. Recommendations for future studies include air sampling around the patient's bedside and identification of bacteria and fungi grown from these samples to establish if similar species are present in nebulisers used within the patient's ventilator circuit. In addition the development and implementation of nebuliser decontamination protocols to investigate their effect on incidence of contamination and incidence of VAP in ICU as no such protocols could be found in the literature.

\section{CONCLUSION}

The majority of nebulisers used within a ventilator circuit were single-use jet nebulisers that were being re-used with more than half being contaminated. All staff including physiotherapists as members of the inter-professional team in ICU should be aware of the medicolegal implications of re-use of single-use nebulisers and be actively involved in the drafting of and implementation of protocols for the decontamination and storage of nebulisers in ICU.

\section{ACKNOWLEDGEMENT}

Many thanks to Associate-Professor Sandy van Vuuren, Pharmaceutical Microbiological Laboratory, Faculty of Health Sciences, University of the Witwatersrand, for her assistance with this project.

\section{REFERENCES}

Allan J, Cunniffe JG, Edwards C, Kretzer D, Ledgerton A, Makintosh C, Murray AE 2004. Nebuliser Decontamination. Journal of Hospital Infection 59(1):72-74.

Anaissie EJ, Penzak SR, Dignani C 2002. The Hospital Water Supply as a Source of Nosocomial Infections: A Plea for Action. Archives of Internal Medicine 162:1483-1492.

Ball C, Cox D, Englebretson K, Hill C, Thacker M 2005. Medical Devices and Their Role in the Incidence of Ventilator Associated Pneumonia Challenging Some Sacred Cows! Intensive and Critical Care Nursing 21(3):131-134.

Bhagwanjee S, Scribante J 2007. National Audit of Critical Care Resources in South Africa - Unit and Bed Distribution. South African Medical Journal 97(12):1311-1314.

Dhand R 2008. Aerosol Delivery During Mechanical Ventilation: From Basic Techniques to New Devices. Journal of Aerosol Medicine and Pulmonary Drug Delivery 21(1):45-60.

Edwards A 2001. Decontamination of nebulisers. http://www.nursingtimes.net/decontamination-ofnebulisers/206122.article [Accessed 20/03/2013].

Fink J 2009. Aerosol Drug Therapy. In: RL Wilkins, JK Stoller, RM Kacmarek (eds) Egan's Fundamentals of Respiratory Care. $9^{\text {th }}$ ed. pp 824 836. Missouri: Mosby Inc.

Robinson BRH, Athota KP, Branson RD 2009. Inhalational Therapies for the ICU. Current Opinion in Critical Care 15:1-9.

Rotstein C, Evans G, Born A, Grossman R, Light RB, Magder S, McTaggert B, Weiss K, Zhanel GG 2008.Clinical Practice Guidelines for Hospital-Acquired Pneumonia and VentilatorAssociated Pneumonia in Adults. Canadian Journal of Infectious Diseases and Medical Microbiology 19(1):19-53.

Sagripanti JL, Grote G, Niederwöhrmeier B, Marschall HJ 2013. Inactivation of Pseudomonas aeruginosa by direct sunlight. Photochemistry Photobiology Feb 27. doi: 10.1111/php.12059. [Epub ahead of print].

Schobert M, Tielen P 2010. Contribution of oxygen-limiting conditions to persistent infection of Pseudomonas Aeruginosa. Future Microbiology 5(4):603-621.

Valencia M, Torres A 2009. Ventilator-Associated Pneumonia. Current Opinion in Critical Care 15:30-35. 